\title{
Exploitation De L'huître Des Mangroves Crassostrea Gasar (Adanson, 1757) Dans Le Lac Nokoué Au Bénin
}

\author{
Hyppolite Agadjihouede \\ David Géraud Akele
}

Ecole d'Aquaculture de la Vallée, Université Nationale d'Agriculture, Bénin

Armel Ulrich Mahounan Gougbedji

Philippe Adédjobi Laleye

Laboratoire d'Hydrobiologie et d'Aquaculture, Faculté des Sciences

Agronomiques, Université d'Abomey-Calavi, Bénin

doi: 10.19044/esj.2017.v13n12p352 URL:http://dx.doi.org/10.19044/esj.2017.v13n12p352

\begin{abstract}
This manuscript deals with the exploitation of oysters, Crassostrea gasar, specie highly presented in Lake Nokoué, south of Benin Republic. This study aims to characterize this exploitation through the sociodemographic parameters of oyster farmers, the characteristics of different collection sites, the importance and economic characteristics of the exploitation. Socio-demographic and economic information was collected on the basis of a survey of the operators. The physico-chemical data and on the type of substrate used to characterize the sites were collected on the stations Agbato and Atchakpanoukpa. A total of 65 farmers were surveyed. In general, women dominate the people who exploit the oysters $(67.7 \%)$. Harvesting and trading are two components of the exploitation of oysters. Harvesting is an essentially masculine activity while trading is reserved to women. The stations Agbato, Donoukpa, Jesukô, Togbigba and Atchakpanoukpa near to Cotonou channel are the harvest sites characterized by high salinity $(24.05 \pm 7.70 \mathrm{~g} / \mathrm{L})$. The oysters' exploitation in Lake Nokoue is realized at march to september of each year by $60 \%$ of populations surveyed. The oysters' exploitation weekly generates incomes of F CFA 30150.77 to the collectors, F CFA 29703.57 to fishmongers and F CFA 28763.33 to whom lead the two activities. Oysters' exploitation is intense at Lake Nokoué and constitutes important source of incomes to the operators.
\end{abstract}

Keywords: Lake Nokoué, Oyster, Crassostrea gasar, Exploitation, Harvesting sites, Benin. 


\section{Résumé}

Ce manuscrit traite de l'exploitation de l'huître Crassostrea gasar, une espèce fortement retrouvée dans le Lac Nokoué au sud-Bénin. L'objectif de ce travail est de caractériser cette exploitation à travers les paramètres socio-démographiques des exploitants d'huîtres, les caractéristiques des différents sites de récolte, l'importance de l'exploitation et ses caractéristiques économiques. Les données socio-démographiques et économiques ont été recueillies sur la base d'une enquête réalisée auprès des exploitants. Les paramètres physico-chimiques et la nature du substrat des sites de récolte ont été collectées sur les stations Agbato et Atchakpanoukpa. $\mathrm{Au}$ total, 65 exploitants ont été enquêtés. Les femmes dominent les populations qui exploitent la ressource $(67,7 \%)$. La récolte et le mareyage sont les deux composantes de l'exploitation de l'huître. La récolte est une activité essentiellement masculine alors que le mareyage est réservé aux femmes. Les stations Agbato, Donoukpa, Jesukô, Togbigba et Atchakpanoukpa situées à proximité du chenal de Cotonou sont les sites de récoltes caractérisés par une forte salinité $(24,05 \pm 7,70 \mathrm{~g} / \mathrm{L})$. L'exploitation se fait de mars à septembre et occupe plus de $60 \%$ de la population enquêtée. Elle génère des revenus moyens hebdomadaires de 30 150,77 Fcfa aux récolteurs, 29 703,57 Fcfa aux mareyeuses et 28 763,33 Fcfa à celles qui mènent les deux activités. L'exploitation de l'huître est intense dans le Lac Nokoué et constitue une source importante de revenus aux exploitants.

Mots-clés : Lac Nokoué, Huître, Crassostrea gasar, Exploitation, Sites de récolte, Revenus.

\section{Introduction}

L'importance des huîtres dans le monde est indéniable tant sur le plan alimentaire (Jouzier, 1998), écologique (Tamburri et al., 2008) qu'économique (Baron, 1992).

Au Bénin les huîtres, communément appelées « Adakpin » en Fongbé et « Atcha » en Aïzo, sont des ressources aquatiques exploitées par la pêche continentale. Elles font l'objet d'une exploitation traditionnelle au Nord du Bénin dans la rivière Pendjari avec l'espèce Etheria elliptica (Akélé et al., 2015). Au Sud du pays, elles sont exploitées au niveau de plusieurs plans d'eau notamment dans le lac Nokoué avec l'espèce Crassostrea gasar (Sènouvo, 2003) connue également sous le nom d'huître des mangroves.

Les palétuviers (Rhizophora racemosa) sont caractéristiques des écosystèmes de mangroves et constituent l'habitat naturel de l'huître de mangrove (Lapègue et al., 2002). Pourtant, l'utilisation de ces arbres pour la construction des « acadjas » et pour divers usages domestiques a dénudé des 
dizaines de kilomètres de mangrove le long des berges. Dans le lac Nokoué, la mangrove a pratiquement disparu (Gnohossou, 2006). La destruction de cet habitat induit inéluctablement une baisse du recrutement naturel des naissains. Outre la contribution sommaire à l'étude de l'huître exploité au lac Nokoué de Abiogba et Henadou en 2006, aucune donnée récente n'existe quant à l'exploitation des huîtres dans le lac Nokoué. Des connaissances actualisées sur le système d'exploitation d'une ressource permettent d'évaluer l'état de cette ressource et d'en effectuer une gestion efficiente. C'est pour cela que la présente étude est réalisée et vise à caractériser le système d'exploitation de Crassostrea gasar dans le lac Nokoué à travers ses dimensions socio-démographique, biologique et économique.

\section{Materiel Et Methodes}

\subsection{Milieu d'étude}

Le lac Nokoué (Figure 1) est situé au Sud-Est du système lagunaire côtier béninois $\left(6^{\circ} 25^{\prime} \mathrm{N}, 2^{\circ} 36^{\prime} \mathrm{E}\right)$. Il constitue l'écosystème saumâtre le plus important du Bénin avec une superficie de $150 \mathrm{~km}^{2}$ (Villanueva, 2004). Il communique à l'Est avec la lagune de Porto-Novo $\left(35 \mathrm{~km}^{2}\right)$ par l'intermédiaire du canal Totchè, long de $5 \mathrm{~km}$ et d'une largeur de $200 \mathrm{~m}$ à $300 \mathrm{~m}$ environ (Gnohossou, 2006). Il est également sous l'influence des débits fluviatiles de l'Ouémé et de la rivière Sô dans sa partie Nord. De même, il s'ouvre directement dans l'Océan Atlantique par le chenal de Cotonou sur une longueur de 4,5 $\mathrm{km}$ avec une largeur de $300 \mathrm{~m}$ environ (Gnohossou, 2006). D'après Lalèyè (1995), le régime hydrologique du lac Nokoué est caractérisé par trois saisons. Une saison " sèche », de décembre à mars, où les apports continentaux sont négligeables, contrairement à l'influence maritime prédominante; la transparence, la température et la salinité atteignent leur valeur maximale au moment où la hauteur d'eau est minimale; une saison " des pluies » d'avril à juillet où les températures atteignent leur minimum et une saison « des crues » d'août à novembre où la salinité devient minimale, le $\mathrm{pH}$ atteint ses valeurs minimales et l'oxygène dissous atteint ses valeurs maximales. 


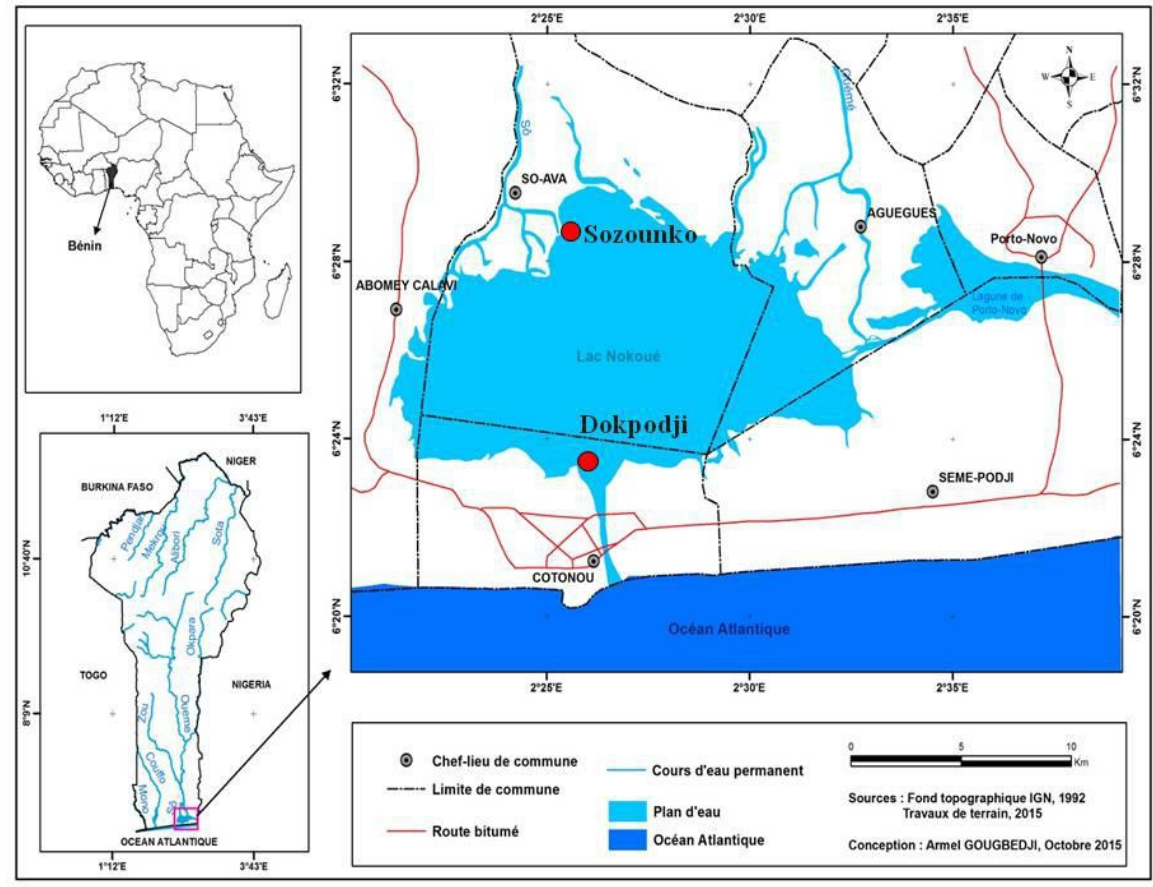

Figure 20. Situation géographique du lac Nokoué.

\subsection{Collecte des données}

Paramètres socio-démographiques des exploitants : La réalisation de la présente étude s'est appuyée sur une enquête systémique auprès des exploitants de l'huître dans le lac Nokoué. Cette enquête s'est déroulée en septembre 2015 dans deux villages, Dokpodji et Sozounko (Figure 1). Ces villages ont été choisis en fonction de l'effectif de la population impliquée dans l'exploitation; respectivement très élevé et moyen.

$>$ Un échantillonnage aléatoire simple a été réalisé au niveau de chaque village dans la mesure où l'on ne disposait pas au préalable d'informations concernant la structure de la population cible. La taille de l'échantillon a été constituée par les exploitants disponibles dans la période d'étude. A Dokpodji, 28 exploitants ont été enquêtés tandis que 37 exploitants ont été questionnés à Sozounko (12 000 habitants). Sur la base d'un guide d'entretien, les données socio-démographiques tels que le sexe, l'âge, les activités menées et celles économiques ayant trait à la commercialisation et aux revenus bruts ont été récoltées au près des exploitants. De même, les raisons du choix de cette activité ont été recueillies et hiérarchisées selon la méthode de distribution des cailloux. 
$>$ Caractéristiques des sites d'exploitation: Par ailleurs, les sites de récolte des huîtres ont été identifiés puis géoréférencés. Afin de caractériser ces sites de récolte, les paramètres physico-chimiques tels que la température, la transparence, le $\mathrm{pH}$, l'oxygène dissous et la salinité ont été mesurés sur 5 mois (Avril-Août 2015) dans deux stations (Agbato et Atchakpanoukpa). De plus le substrat a été prélevé dans les deux stations.

Exploitation: Pour caractériser l'exploitation de l'huître, 10 spécimens de toutes les tailles ont été récoltés dans les deux stations pendant 5 mois. Pour chaque spécimen prélevé, la hauteur (distance entre l'umbo et la base de la région postérieur) et le poids ont été mesurés respectivement au moyen d'un pied à coulisse et d'une balance électronique afin d'évaluer les tailles moyennes et les biomasses prélevées.

\subsection{Traitement des données}

Des histogrammes ont été tracés afin d'analyser les caractéristiques des exploitants de la ressource en fonction de l'âge, du sexe et du niveau d'instruction. Les structures d'âges ont été tracées après regroupement des classes d'âges en jeune $([18 ; 35])$, adulte $([36 ; 55])$ et vieux $(\geq 56$ ans) selon Petry (2002).

Par ailleurs, les exploitants enquêtés ont été catégorisés en récolteurs; mareyeuses et mixtes (à la fois récolteurs et mareyeuses). A partir du géoréférencement des sites de récoltes, une carte de distribution de l'huître dans le lac a été réalisée. L'importance de l'exploitation a été évaluée par la proportion de la population exerçant l'activité dans chaque village, les périodes de récolte et la fréquence de récolte.

Les caractéristiques économiques de l'exploitation de l'huitre ont été déterminées par le calcul des revenus bruts hebdomadaires.

Des tests de comparaison multiple de proportions ont été réalisés entre les villages pour les paramètres socio-démographiques; le test de Mann-Whitney a été effectué pour comparer les stations du point de vue physico-chimie, les villages du point de vue niveau d'exploitation et les revenus entre les catégories d'exploitants. Le test de concordance de Kendall a été utilisé pour évaluer le degré de consensus entre les exploitants quant aux raisons du choix de l'activité.

\section{Resultats}

\subsection{Caractéristiques des acteurs}

\subsubsection{Caractéristiques Socio-démographiques}

$\mathrm{Au}$ total, 65 exploitants ont été enquêtés dans les deux villages: 28 exploitants à Dokpodji et 37 à Sozounko. Les Toffins représentent la seule 
ethnie identifiée au cours de l'enquête. La figure 2-A présente les différentes catégories d'âge exploitant la ressource par village. Dans le village de Dokpodji, les jeunes et les adultes constituent respectivement $42,86 \%$ et $39,29 \%$ de l'effectif enquêté. Les vieux représentent $17,86 \%$ de la population cible. En ce qui concerne Sozounko, les jeunes sont majoritaires avec une proportion de $43,24 \%$ des personnes questionnées. Les adultes viennent ensuite avec $35,14 \%$ et enfin les vieux sont représentés à $21,62 \%$. Que ce soit pour les populations enquêtées de jeunes, d'adultes et de vieux, le test de comparaison multiple des proportions n'a révélé aucune différence significative entre les deux localités $(p>0,05)$.

Dans les deux villages, la population d'exploitant est dominée par les femmes (Figure 2-B). En effet, elles représentent 89,29\% des exploitants de Dokpodji et $51,35 \%$ de ceux de Sozounko. Il existe une différence significative entre les proportions des femmes exploitantes de l'huitre de ces villages $(p<0,05)$. Il en est de même pour les individus du sexe masculin.
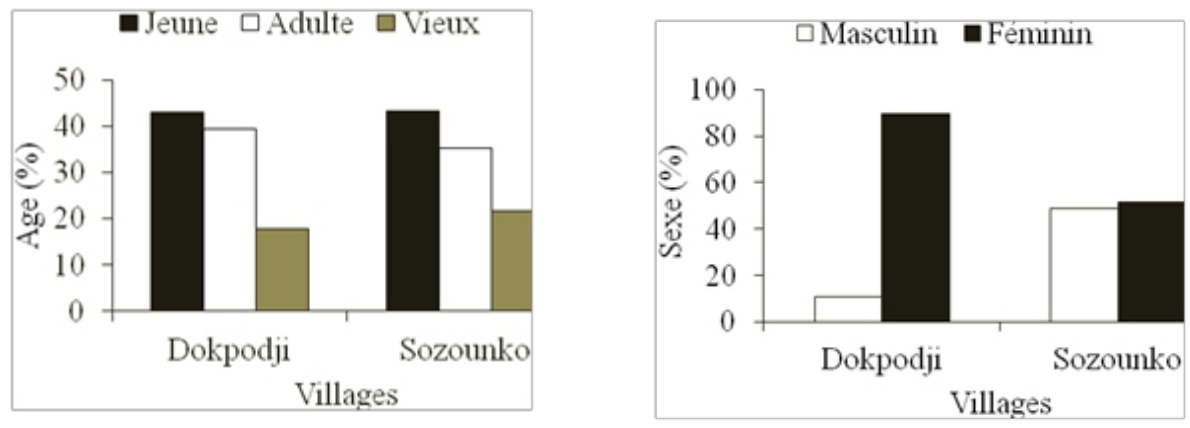

Figure 21. Caractéristiques socio-démographiques des enquêtés. A: Selon l'âge; B: Selon le sexe.

\subsubsection{Statut de récolteur ou de mareyeuse}

Dans les deux villages où l'enquête s'est déroulée, $100 \%$ des hommes interrogés sont des récolteurs; aucun d'eux ne se livre aux activités de mareyage ou de transformation de l'huître (Figure 3). Parmi les femmes enquêtées à Dokpodji, $48 \%$ d'entre elles s'adonnent aussi bien à la récolte qu'au mareyage de l'huître (activité mixte). L'activité exclusive de mareyage de l'huître est menée par $44 \%$ des femmes et les récolteurs de sexe féminin sont de $8 \%$. Par contre, la majorité $(68,18 \%)$ des femmes questionnées à Sozounko sont exclusivement des mareyeuses. Les activités simultanées de récolte et de mareyage sont menées par $13,64 \%$ des femmes alors que de récolte seule est pratiquée par 5,26\% des femmes. Il existe une différence significative entre les proportions des statuts mareyeuse et mixte des deux villages; $(\mathrm{p}<0,05)$. 


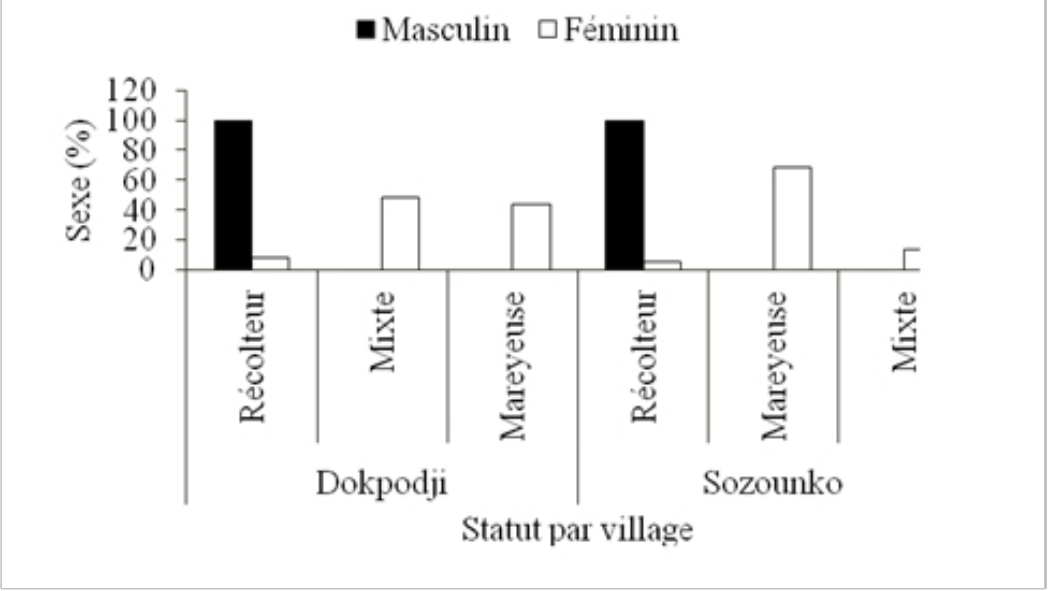

Figure 22. Proportion des enquêtés par statut et par village.

\subsubsection{Activités génératrices de revenus des acteurs}

La figure 4 présente les activités recensées comme génératrices de revenus dans les deux villages. Au total, quatre activités ont été identifiées; il s'agit de l'exploitation de l'huître, du commerce, des activités de pêche et d'autres activités sources de revenus (Exploitation de carrières, vente d'essence, exploitation d'Acadjas, etc.).

Dans les deux localités, l'activité principale durant toute l'année est l'exploitation de l'huître ( $100 \%$ des enquêtés). L'activité secondaire est le commerce; que ce soit à Sozounko $(37,84 \%)$ ou à Dokpodji $(35,71 \%)$. L'activité de pêche occupe la troisième place à Dokpodji (25\%) alors qu'elle est en dernière position à Sozounko $(8,11 \%)$. Les autres activités sont menées par $10,81 \%$ des enquêtés de Dokpodji et par $7,14 \%$ de ceux de Sozouko. Aucune différence significative n'a été enregistrée entre les deux villages pour chaque activité $(p>0,05)$.

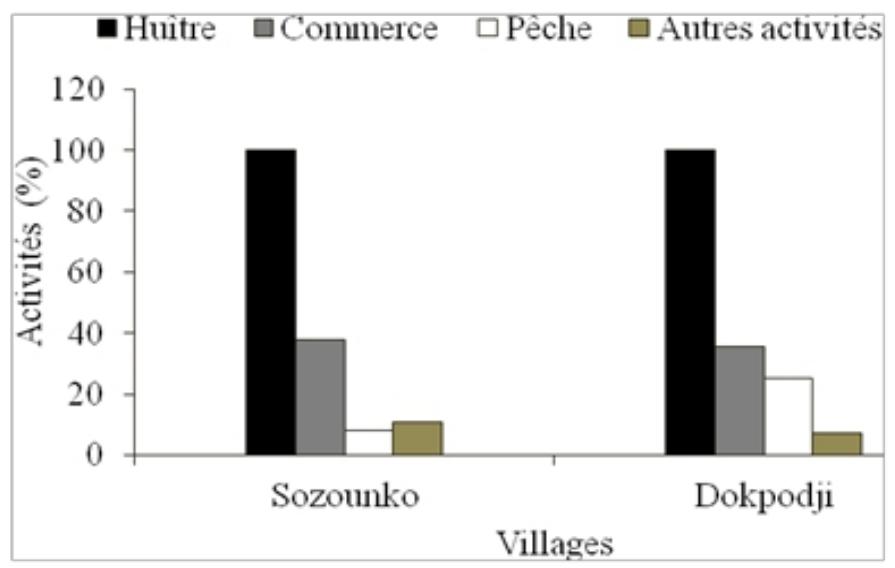

Figure 23. Proportion des activités génératrices de revenues. 


\subsubsection{Motivations sous-tendant l'exploitation de l'huître dans le lac Nokoué}

Cinq motivations qui justifient le choix de l'activité de récolte d'huître ou de mareyage ont été recensées (Tableau 1). Il s'agit de:

> Activité traditionnelle: Cette raison indique que l'exploitation de l'huître est une activité qui se transmet d'une génération à l'autre. Elle constitue la première compétence professionnelle acquise par l'exploitant.

$>$ Complément de revenus: L'exploitation de l'huître constitue une activité secondaire qui sert à augmenter de façon substantielle le revenu généré par l'activité principale.

$>$ Rareté du poisson et disponibilité de l'huître: L'activité de récolte ou de mareyage de l'huître constitue une alternative à la diminution du stock ichtyologique.

> Rentabilité: L'exploitation de l'huître constitue une source de revenu très importante.

$>$ Revenus de subsistance: L'activité permet de subvenir, dans la limite du possible aux besoins d'entretien.

La raison première du choix de l'exploitation de l'huître évoquée par 43,08\% des enquêtés est la rentabilité qu'elle procure. La deuxième motivation est le complément de revenus qui est classée dans ce rang par 29,23\% des exploitants. Les revenus de subsistance que procurent l'activité est la troisième raison évoquée par $24,62 \%$ des enquêtés. Le caractère traditionnel de l'activité constitue le quatrième choix de la population $(27,69 \%)$ et $29,23 \%$ des exploitants sont unanimes sur le dernier rang représenté par la rareté du poisson et la disponibilité de l'huître.

Le tableau 2 présente les résultats du test de concordance de Kendall. Le coefficient de concordance de Kendall obtenu $(\mathrm{W}=0,2)$ révèle un faible niveau de consensus dans les raisons qui motivent le choix de l'exploitation de l'huître. Par ailleurs, la statistique de $\chi^{2}$ associée est hautement significative $(\mathrm{p}<0,05)$.

Tableau 2. Hiérarchisation des motivations qui sous-tendent l'exploitation de l'huître.

\begin{tabular}{|l|l|l|l|l|l|}
\hline \multicolumn{1}{|c|}{ Rangs } & $\mathbf{1}$ & $\mathbf{2}$ & $\mathbf{3}$ & $\mathbf{4}$ & $\mathbf{5}$ \\
\hline Mentivations & $\mathbf{4 3 , 0 8}$ & 12,31 & 18,46 & 20,00 & 6,15 \\
\hline Complément de revenus & 35,38 & $\mathbf{2 9 , 2 3}$ & 24,62 & 7,69 & 3,08 \\
\hline Revenus de subsistance & 9,23 & 16,92 & $\mathbf{2 4 , 6 2}$ & 18,46 & 30,77 \\
\hline Activité traditionnelle & 9,23 & 23,08 & 9,23 & $\mathbf{2 7 , 6 9}$ & 30,77 \\
\hline Rareté du poisson et disponibilité de l'huître & 3,08 & 18,46 & 23,08 & 26,15 & $\mathbf{2 9 , 2 3}$ \\
\hline
\end{tabular}

Tableau 3. Résultats du test de concordance de Kendall.

\begin{tabular}{|l|l|}
\hline Paramètres & Valeurs \\
\hline $\mathrm{k}$ & 65 \\
\hline $\mathrm{N}$ & 5 \\
\hline
\end{tabular}




\begin{tabular}{|l|l|}
\hline $\mathrm{W}$ & 0,20 \\
\hline$\chi^{2}$ & 51,13 \\
\hline $\mathrm{p}$ & $<0,000$ \\
\hline
\end{tabular}

\subsection{Caractéristiques de sites de récoltes de l'huître dans le lac Nokoué} 3.2.1. Localisation géographique

La figure 5 représente la répartition spatiale des sites de récolte de l'huître des mangroves dans le lac Nokoué. Tous les sites identifiés sont localisés non loin du chenal de Cotonou qui communique avec l'océan atlantique.

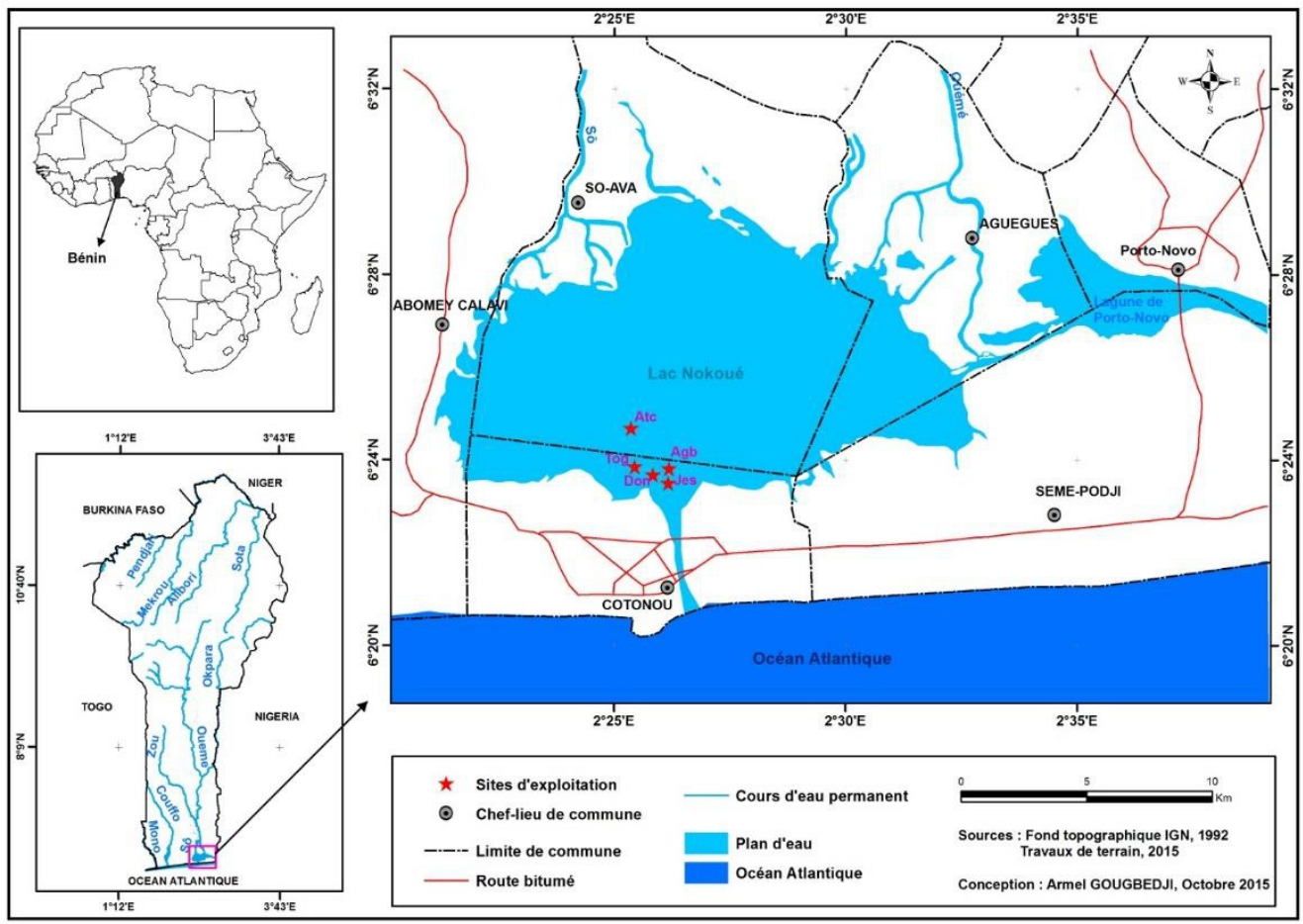

Figure 24. Répartition spatiale des sites de récolte de l'huître dans le lac Nokoué. Agb: Agbato; Don: Donoukpa; Jes: Jesukô; Tog: Togbigba.

\subsubsection{Caractéristiques physico-chimiques et nature du substrat des sites de récolte}

Les valeurs moyennes des paramètres physico-chimiques enregistrées par station sont consignées dans le tableau 3. Les valeurs de la température de l'eau, du $\mathrm{pH}$ de l'eau, de l'oxygène dissous et de la transparence au Secchi n'ont pas varié entre les deux stations (test de Mann-Withney, $\mathrm{p}>$ $0,05)$. Par contre, il existe une différence significative entre les valeurs de l'oxygène dissous des deux stations $(\mathrm{p}<0,05)$.

L'examen macroscopique de l'échantillon des substrats prélevés sur le sédiment en a révélé deux types. Il s'agit d'un substrat sableux et d'un 
substrat vaseux. Le substrat de type sableux a été identifié à Agbato alors que celui du type vaseux a été relevé à Atchakpanoukpa.

Tableau 4. Synthèse des paramètres physico-chimiques de l'eau.

\begin{tabular}{|l|l|l|l|l|l|l|}
\hline \multirow{4}{*}{ Station } & & $\begin{array}{l}\text { Tempér } \\
\text { ature } \\
\left({ }^{\circ} \mathbf{C}\right)\end{array}$ & $\mathbf{p H}$ & $\begin{array}{l}\mathbf{O}_{2} \\
(\mathbf{m g} / \mathbf{l})\end{array}$ & $\begin{array}{l}\text { Salinité } \\
(\mathbf{g} / \mathbf{l})\end{array}$ & $\begin{array}{l}\text { Transpare } \\
\text { nce au } \\
\text { Secchi } \\
(\mathbf{c m})\end{array}$ \\
\hline \multirow{5}{*}{ Agbato } & Moyenne & 26,55 & 7,23 & 5,61 & 26,70 & 85,20 \\
\cline { 2 - 7 } & Ecart-type & 1,85 & 0,30 & 0,40 & 9,05 & 25,29 \\
\cline { 2 - 7 } & CV(\%) & 0,07 & 0,04 & 0,07 & 0,34 & 0,30 \\
\cline { 2 - 7 } & Minimum & 24,55 & 6,91 & 5,13 & 13 & 60 \\
\cline { 2 - 7 } & Maximum & 29,25 & 7,73 & 6,07 & 38 & 121 \\
\hline \multirow{5}{*}{ Atchakpanoukpa } & Moyenne & 26,88 & 7,40 & 5,04 & 21,40 & 85,20 \\
\cline { 2 - 7 } & Ecart-type & 2,15 & 0,28 & 0,07 & 5,81 & 23,86 \\
\cline { 2 - 7 } & CV(\%) & 0,08 & 0,04 & 0,02 & 0,27 & 0,28 \\
\cline { 2 - 7 } & Minimum & 24,15 & 7,06 & 4,94 & 12 & 55 \\
\cline { 2 - 7 } & Maximum & 29,90 & 7,72 & 5,13 & 26 & 112 \\
\hline & Moyenne & 26,72 & 7,31 & 5,33 & 24,05 & 85,20 \\
\cline { 2 - 7 } & Ecart-type & 1,90 & 0,29 & 0,40 & 7,70 & 23,18 \\
\hline
\end{tabular}

\subsection{Caractéristiques de l'exploitation de l'huître dans le lac Nokoué}

\subsubsection{Période et moyens d'exploitation des huîtres}

L'exploitation de l'huître dans le lac Nokoué dure 6 mois. Elle débute en Mars et amorce sa fin à partir du mois de septembre.

Les instruments ou engins utilisés dans l'activité sont rudimentaires et constitués: d'une pirogue monoxyle, d'un bidon de contenance 50 litres découpé sur l'une des faces latérales et muni d'une corde, de chaussettes et de gants. La pirogue sert de moyen de déplacement et de transport de l'huître mais constitue également un instrument de mesure pour la vente de l'huître.

Elle constitue, selon sa taille, l'unité de mesure dans les échanges commerciaux. Le bidon est utilisé pour transporter les huîtres récoltées dans la pirogue. Les chaussettes et les gants servent à protéger le récolteur des blessures occasionnées par les coquilles d'huître.

\subsubsection{Techniques d'exploitation des huîtres}

La technique de récolte (Figure 6-A) est relativement simple. Le récolteur attache la corde du bidon autour de sa taille puis descend dans l'eau. Avec ses pieds, il repère les colonies d'huîtres sur le substrat puis plonge sous l'eau. Une fois au fond, il ramasse avec ses mains les huîtres qu'il ramène dans le bidon qui flotte en surface de l'eau. Une fois le bidon rempli, son contenu est déversé dans la pirogue (Figure 6-B).

La fréquence moyenne de récolte de l'huître dans les deux villages est de 5 jours et la durée d'une récolte est évaluée à 9 heures. La taille.. 
moyenne des huîtres vendues au cours des cinq mois d'exploitation est de $6,7 \mathrm{~cm}$.

L'huître récoltée est vendue aux femmes qui procèdent à la transformation du produit (Figure 7). La première étape consiste à bouillir l'animal avec sa coquille dans une marmite pendant 3 à 4 heures de temps. Après la cuisson, la mareyeuse, munie d'un couteau enlève la chair de l'animal et fait un stock des coquilles. La chair qui est la partie consommée de l'animal est mise en tas pour la vente. La fréquence moyenne de mareyage est de 4 jours pour les deux villages et la durée moyenne de cette activité est de 12 heures par jour.
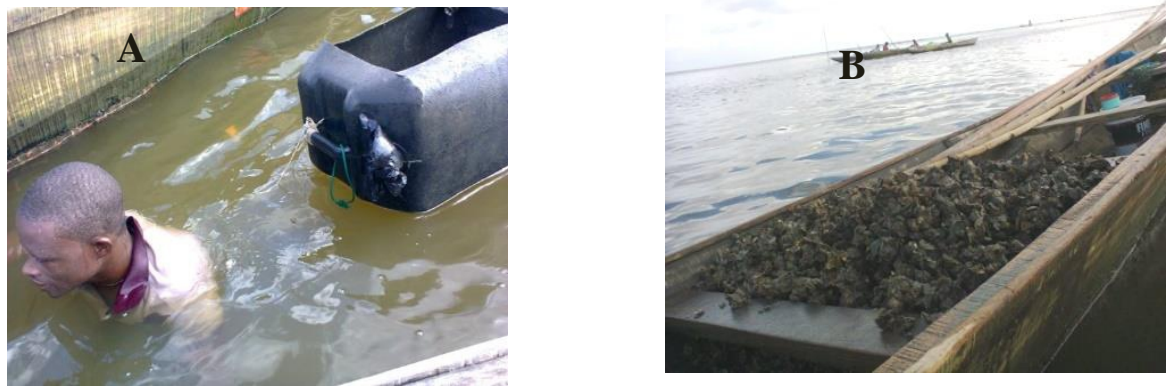

Figure 25. Récolte de l'huître dans le lac Nokoué. A: Technique de récolte de l'huître; B: Pirogue contenant des spécimens d'huîtres récoltés.
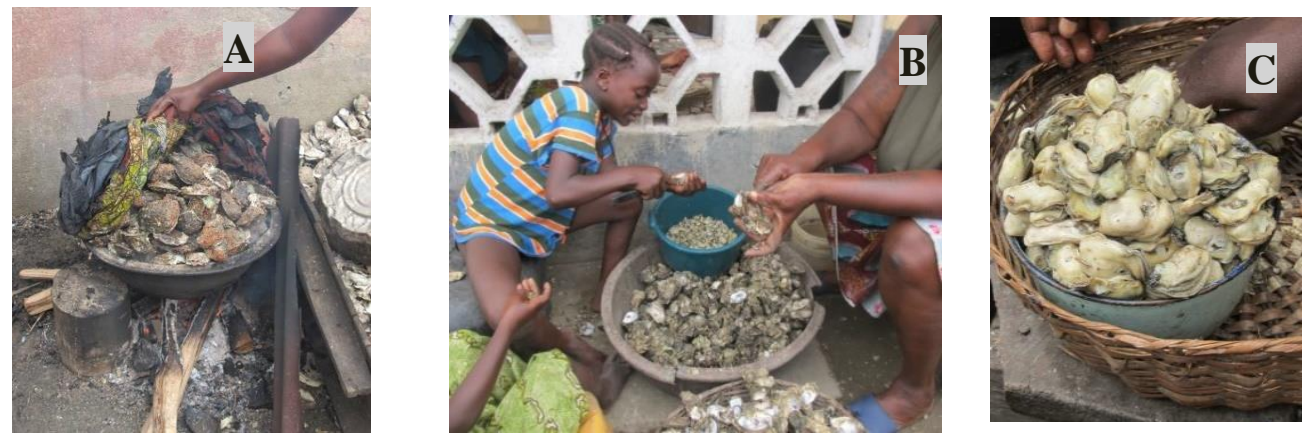

Figure 26. Préparation de l'huître pour la vente. A: Cuisson; B: Enlèvement de la chair; C: Mise en tas pour la vente.

\subsubsection{Caractéristiques économiques de l'exploitation de l'huître}

Le tableau 4 présente les prix de vente des huîtres selon les mesures. Les unités de mesures sont les pirogues dont les dimensions ont permis de classer en trois catégories: Petit, Moyen et Grand. Les prix de vente sont proportionnels à la taille des pirogues. Ces huîtres sont vendues aux femmes qui vivent dans la localité de résidence du récolteur.

Les quantités récoltées par sortie varient de 170 à $284 \mathrm{~kg}$ pour les petites pirogues; 340 à $455 \mathrm{~kg}$ pour les pirogues moyennes et 567 à $682 \mathrm{~kg}$ pour les grandes pirogues. 
Les prix de vente de l'huître transformé varient selon la forme finale du produit. Ainsi, le prix du kilogramme de la chair de l'huître crue varie ente 1000-1200 F CFA (Tableau 5). Les huîtres frites sont embrochées par lot de 8 ou 10 (selon la taille) et vendu à 100 F CFA la brochette. L'huître est vendue à domicile ou au marché international Dantokpa de Cotonou. Par ailleurs, les coquilles conservées après chaque transformation sont stockées dans des sacs et vendues à raison de $400 \mathrm{~F} \mathrm{CFA} \mathrm{ou} 500 \mathrm{~F}$ CFA le sac. Les acheteurs usuels de ces coquilles sont les centres de production d'aliments pour animaux.

Les revenus bruts moyens que génère l'exploitation de l'huître par semaine sont présentés dans le tableau 6. Les récolteurs sont ceux dont les revenus sont les plus élevés. Les mareyeuses viennent en deuxième position et les personnes qui mènent les deux activités ont les plus faible revenus. Le test de Mann-Whitney a révélé une différence significative entre les revenus des récolteurs et les revenus de ceux qui mènent les deux activités $(p<0,05)$.

Tableau 5. Prix de vente de l'huître récoltée par unité de mesure.

\begin{tabular}{|c|c|c|c|c|c|}
\hline $\begin{array}{c}\text { Type de } \\
\text { pirogue }\end{array}$ & $\begin{array}{c}\text { Longueur } \\
(\mathbf{m})\end{array}$ & $\begin{array}{c}\text { Largeur } \\
(\mathbf{m})\end{array}$ & $\begin{array}{c}\text { Hauteur } \\
(\mathbf{c m})\end{array}$ & $\begin{array}{c}\text { Quantité } \\
\text { prélevée } \\
(\mathbf{k g})\end{array}$ & $\begin{array}{c}\text { Prix moyen } \\
(\text { FCFA) }\end{array}$ \\
\hline Petit & 6,5 & 0,97 & 34 & $170-284$ & 5000 \\
\hline Moyen & 7,5 & 1,25 & 37 & $340-455$ & 7923,08 \\
\hline Grand & 8,5 & 1,6 & 40 & $567-682$ & 13333,33 \\
\hline
\end{tabular}

Tableau 6. Prix de vente de l'huître selon la forme de la transformation.

\begin{tabular}{|c|c|c|}
\hline Forme du produit & Mesure & Prix (FCFA) \\
\hline Cru & Bol $(1 \mathrm{~kg})$ & $1000-1200$ \\
\hline Frit & Brochettes de 10 & 100 \\
\hline
\end{tabular}

Tableau 7. Revenus bruts moyens hebdomadaires générés par l'exploitation de l'huître.

\begin{tabular}{|c|c|}
\hline Statut & Revenus hebdomadaire (FCFA) \\
\hline Récolteur & 30150,77 \\
\hline Mareyeuse & 29703,57 \\
\hline Récolteur et mareyeuse & 28763,33 \\
\hline
\end{tabular}

\section{Discussion}

L'exploitation de l'huître des mangroves $C$. gasar dans le lac Nokoué implique une population importante dont 1'échantillon enquêté est constitué d'un seul groupe ethnique: les Toffins. L'un des traits Socio-démographiques très important qui caractérise les exploitants d'huître est la forte proportion des femmes parmi les personnes questionnées. Ce constat a déjà été fait à la lagune côtière au Bénin (Kinkpé et al., 2005) où l'exploitation de l'huître des mangroves est l'apanage du genre féminin. De même, l'exploitation de 
l'huître des rivières Etheria elliptica à la rivière Pendjari est dominée par les femmes (Akélé et al., 2015). En casamance au Sénégal, l'exploitation des huîtres depuis la cueillette jusqu'à la vente est une activité exclusivement féminine (IDEE Casamance, 2005).

Des deux villages enquêtés, c'est celui de Dokpodji qui compte une proportion plus élevée de femmes exploitantes. Le sexe masculin semble peu intéressé par l'activité dans le village de Dokpodji alors que la population masculine de Sozounko est fortement représentée parmi les exploitants. Il est fort probable que cette implication très faible des hommes de Dokpodji soit due à la situation géographique du village. En effet, il est situé dans la zone du chenal de Cotonou (Figure 1) qui communique avec la mer. La diversité de la faune ichtyologique y est élevée (Niyonkuru, 2007) dans la mesure où le milieu connait la migration de plusieurs espèces d'origine marine. Cette potentialité de la pêche pourrait donc être un meilleur atout pour les hommes qui y résident. De plus Dokpodji est dans la commune de Cotonou. Sa proximité avec la ville de Cotonou favorise les échanges commerciaux, augmente les possibilités d'emploi et la réalisation d'autres activités. De même, le marché Dantokpa qui a été déterminé comme le principal point de vente de l'huître pour les mareyeuses est très proche du village. Ceci pourrait expliquer la prépondérance des femmes de Dokpodji par rapport à celles de Sozounko.

De façon globale, ce sont les jeunes et les adultes qui s'adonnent à l'activité de récolte ou de mareyage. Ce résultat est conforme à celui d'Akélé (2015) qui a identifié une moyenne d'âge de 40 ans chez les exploitants de la zone libre de la rivière Pendjari. Ceci s'explique aisément, dans la mesure où l'exploitation de l'huître est un travail pénible, long et fatigant (IDEE Casamance, 2005).

L'exploitation de l'huître dans le lac Nokoué revêt deux aspects qui en font une particularité. L'activité comprend deux volets; celui de la récolte et celui du mareyage. La présente étude a révélé que les activités de récolte sont largement dominées par les hommes et que le mareyage est exclusivement réservé aux femmes. Ce partage des rôles est différent des observations réalisées à la lagune côtière (Kinkpé et al., 2005), à la rivière Pendjari (Akélé et al., 2015) et en Casamance au Sénégal (IDEE Casamence, 2005). En effet, l'exploitation de l'huître dans ces régions connait très peu l'intervention des hommes du milieu. La cause probable de cette spécialisation est la contrainte physique inhérente à l'activité de récolte au lac Nokoué. En effet, la méthode de récolte qui consiste à plonger sous l'eau pour récolter l'huître nécessite un déploiement de force d'une intensité et d'une fréquence sans doute limitant pour le sexe féminin. Par contre à la lagune côtière, des instruments rudimentaires permettent de décoller les huîtres des racines échasses de palétuviers. De plus le lac Nokoué est un 
milieu saumâtre; l'ouverture des yeux sous l'eau afin de repérer les bands d'huîtres constitue également un handicape majeur qui est cependant absent à la rivière Pendjari.

Cinq principaux sites de récoltes ont été identifiés dans le lac Nokoué. Il s'agit de Agbato, Donoukpa, Jesukô, Togbigba et Atchakpanoukpa. La répartition spatiale de ces sites montre qu'ils sont situés à proximité du chenal de Cotonou. Or ce chenal communique avec l'océan atlantique, ce qui fait de cette zone un milieu à forte salinité comme le montre les mesures effectuées. Les sites de récoltes répondent donc bien aux conditions de développement des huîtres dans la mesure où la salinité est un paramètre déterminant dans la distribution de l'huître des mangroves (Gosling, 2003). La destruction de la mangrove dans le lac Nokoué (Gnohossou, 2006) a induit une adaptation de C. gasar qui a perdu son habitat naturel. En effet, l'huître se fixe sur des coquilles de spécimens morts retrouvés dans les sédiments sableux et vaseux. Cette adaptation caractérisée par l'utilisation de substrats alternatifs a déjà été observée par Zabi et Le Loeuff (1992).

Le premier critère de l'évaluation de l'importance de l'exploitation est la proportion de la population impliquée dans l'activité. Dans les deux villages enquêtés, elle est plus de $60 \%$ de la population et démontre ainsi de l'intérêt porté à l'exploitation de l'huître dans le lac Nokoué. De même, La fréquence de récolte qui est de 5 jours en moyenne et de mareyage qui est de 4 jours démontre bien du temps consacré à l'activité. Cette observation est d'autant plus vraie que la hiérarchisation des motivations montre que la rentabilité de l'activité est la motivation première de son choix. Le faible niveau de consensus révélé par le test de Kendall serait du à l'inexistence de différence entre les proportions des autres motivations $(p>0,05)$.

L'exploitation de l'huître dans le lac Nokoué dure 6 mois; elle commence en Mars et fini en septembre. Cette période correspond à celle de reproduction et de croissance de $C$. gasar telle que identifiée par Diadhiou (1995). Un autre critère de l'importance de cette activité est le revenu hebdomadaire brut enregistré au niveau des exploitants. Les récolteurs ont un revenu hebdomadaire moyen de 30 150,77 Fcfa. Le revenu des mareyeuses est de 19 703,57 Fcfa et celui des récolteurs-mareyeuses est de 28 763,33 Fcfa. Ces revenus sont supérieurs à ceux enregistrés par Akélé et al. (2015), qui varient de 44000 à $77000 \mathrm{f}$ par saison de récolte au niveau de la rivière Pendjari. Ceci démontre l'envergure de l'exploitation et pourrait justifier le fait que cette activité, qui ne dure que la moitié de l'année, soit cependant la principale enregistrée chez les enquêtés. 


\section{Conclusion}

Cette étude réalisée sur l'exploitation de l'huître des mangroves a permis de caractériser cette activité au lac Nokoué. Ladite activité connait une forte implication des femmes qui y trouvent une source importante de revenus. Une autre caractéristique essentielle de l'exploitation des huitres dans le lac Nokoué est la spécialisation des hommes dans l'activité de récolte et celle des femmes dans le mareyage. Quelques femmes, cependant, bravent les difficultés associées à l'activité de récolte et mènent les deux activités. Elles augmentent ainsi leur marge bénéficiaire. Chaque année, la saison de récolte débute en mars et prend fin en septembre. Cette période correspond aux conditions de salinité élevé dans le lac. Agbato, Donoukpa, Jesukô, Togbigba et Atchakpanoukpa sont les cinq sites de récoltes qui ont été identifiés au terme de l'étude. Ces sites, caractérisés par une salinité élevée sont des biotopes de prédilection pour les huîtres qui ne vivent que dans les milieux mésohalins.

\section{Remerciements}

Les auteurs tiennent à remercier Dr AOUDJI Augustin, Agro-socioéconomiste pour ses orientations dans la collecte et le traitement des données et tous les examinateurs anonymes pour leur contribution à l'amélioration de la qualité scientifique de ce manuscrit.

\section{References:}

1. Akélé, G.D., et al., 2015. Traditional exploitation of edible freshwater oyster Etheria elliptica (Lamarck, 1807) in Pendjari River (BeninWest Africa): assessment of income, human pressure and options for management. International Journal of Biological Chemical Science. 9(1), 246-258.

2. Baron, J., (1992) Reproductive cycles of the Bivalve Molluscs Atactodea striata (Gmelin), Gafrarium tumidum and Anadra scapha (L.) in New Caledonia. Australien Journal of Marine Freshwater Research 43, 393-402.

3. Diadhiou, H.D., (1995) Biologie de l'huître de palétuvier Crassostrea gasar (Dautzenberg) dans 1'estuaire de la Casamance (Sénégal) : Reproduction, larves et captage du naissain. Thèse de doctorat: Université de Bretagne Occidentale (Angleterre).

4. Gnohossou, P.M., (2006) La faune benthique d'une lagune ouest africaine (le lac Nokoué au Bénin), diversité, abondance, variations temporelles et spatiales, place dans la chaine trophique. Thèse de doctorat: Institut National Polytechnique de Toulouse (France).

5. Gosling, E., (2003) Bivalve Molluscs: Biology, Ecology and Culture. Blackwell, 443 p. 
6. IDEE Casamance. 2005. Vade-mecum de l'élevage de l'huître Crassostrea gasar en Casamance. Document technique. 24 p.

7. Jouzier, E., (1998) L'huître: Aliment ou Médicament? Bulletin de la Société de Pharmacie de Bordeaux, 137, 71-89.

8. Kinkpé, R., Sossa, G.N., \& Viaho, C.C., 2005. L’ostréiculture traditionnelle: état des lieux et perspectives d'amélioration. Mémoire pour l'obtention du Diplôme d'Etude Agricole Tropicale (Bénin).

9. Lalèyè, A.P., (1995) Ecologie comparée de deux espèces de Chrisisthys, poissons siluriformes (Claroteidae). Thèse de doctorat : Université de Liège (Belgique).

10. Lapègue, S. et al., 2002. Trans-Atlantic distribution of mangrove oyster species revealed by $16 \mathrm{~S}$ mtDNA and karyological analyses. Biol Bull, 202, 232-242.

11. Niyonkuru, C., (2007) Etude comparée de l'exploitation et de la démographie des poissons Cichlidés dans les lacs Nokoué et Ahémé au Bénin. Thèse de Doctorat : Université d'Abomey-Calavi (Bénin).

12. Petry, N.M., (2002) A comparison of Young, Middle-Age, and Older Adult Treatment-Seeking Pathological Gamblers. The gerontologist, 42(1), 92-99.

13. Sénouvo, P., (2003) Etude de l'impact des pollutions en métaux lourds $(\mathrm{Pb}, \mathrm{Cu}$ et $\mathrm{Zn})$ sur l'écologie de l'huître Crassostrea gasar en zones lagunaires de Cotonou (Bénin) In Livres des résumés. Poissons et pêches Africains: Diversité et utilisation. Troisième Conférence Internationale de l'Association Pana-Africaine des pêches. Cotonou, Bénin, $211 \mathrm{p}$.

14. Tamburri, M.N., Luckenbach, M.W., Breitburg, D. \& Bonniwell, S., (2008). Settlement of Crassostrea ariakensis larvae: effects of substrate, biofilms, sediment and adult chemical cues. Journal of Shellfish Research, 27(3), 601-608.

15.Zabi, G.S.F. \& Le Loeuff, P., (1992) Revue des connaissances sur la faune benthique des milieux margino-littoraux d'Afrique de l'Ouest, Première partie : biologie et écologie des espèces. Revue d'Hydrobiologie Tropicale 25, 209-251. 